\title{
Noninvasive Imaging of Myocyte Apoptosis Following Application of a Stem Cell-Engineered Delivery Platform to Acutely Infarcted Myocardium
}

\author{
Amandine F.G. Godier-Furnémont ${ }^{1}$, Yared Tekabe ${ }^{2}$, Maria Kollaros ${ }^{2}$, George Eng ${ }^{1}$, Alfredo Morales ${ }^{2}$, \\ Gordana Vunjak-Novakovic ${ }^{2}$, and Lynne L. Johnson ${ }^{2}$ \\ ${ }^{I}$ Department of Biomedical Engineering, Columbia University Medical Center, New York, New York; and ${ }^{2}$ Department of Medicine, \\ Columbia University Medical Center, New York, New York
}

The cardioprotective effects of mesenchymal stem cells (MSCs) include reducing myocyte apoptosis, and this effect can be enhanced by preconditioning and encapsulation in a fibrin scaffold. This study aimed to test the hypothesis that apoptosis imaging can detect the cardioprotective effects of a conditioned MSC patch grafted in a rat model of acute myocardial infarction. Methods: Cell culture experiments simulating engraftment of fibrin patches onto beating rat ventricular myocytes exposed to hypoxia showed an effect of conditioned cells to reduce apoptosis. Twenty-three nude rats underwent successful left anterior descending coronary artery occlusion and were divided into 3 groups: transforming growth factor $\beta 1$-conditioned human MSC-laden patches (CP), infarct alone without patch (no patch [NP]), and patch alone (patch only [PO]). Twenty-four hours after myocardial infarction, all rats were injected with 99mTc-hydrazinonicotinamide (99mTc-HYNIC) annexin $\mathrm{V}$ and ${ }^{201} \mathrm{TI}$ and underwent dual-isotope SPECT/CT imaging. Six rats were sacrificed for histology and counting. The remaining rats ( $n=17 ; 1$ rat was eliminated) were injected and imaged on day 7; of those, 3 rats were sacrificed for histology and counting, and the remaining 13 rats survived to day 21 , when they were sacrificed for histology. Numbers of rats imaged on day 7 in the 3 groups were 7 in the CP group, 5 in the NP, and 5 in the PO. Perfused myocardium, infarct size, and 99mTc-HYNIC annexin $\mathrm{V}$ uptake were quantified from the scans from days 1 and 7. ${ }^{99 m}$ Tc-HYNIC annexin V uptake was correlated with quantitative caspase staining, and infarct size as percentage fibrosis was quantified at day 21 . Results: ${ }^{99 m T c-H Y N I C ~}$ annexin $\mathrm{V}$ uptake as percentage injected dose $\left(\times 10^{-4}\right)$ decreased between days 1 and 7 by $1.04 \pm 0.28$ in the CP group, $0.44 \pm 0.17$ in the NP group, and $0.34 \pm 0.27$ in the $\mathrm{PO}$ group $(P=0.003$ for NP vs. $\mathrm{CP}, P=0.005$ for $\mathrm{PO}$ vs. $\mathrm{CP}$, and $P=0.5$ for NP vs. CP). The changes in defect size as percentage myocardium between days 1 and 7 were $-8.83 \pm$ 4.40 in the CP group, $+1.00 \pm 2.24$ in the NP group, and $-0.50 \pm$ 4.20 in the $\mathrm{PO}$ group $(P=0.003$ for NP vs. $\mathrm{CP}, P=0.005$ for $\mathrm{PO}$ vs. $\mathrm{CP}$, and $P=0.50$ for NP vs. PO). ${ }^{99 m T c-H Y N I C}$ annexin $\mathrm{V}$ uptake as percentage left ventricle by scanning correlated

\footnotetext{
Received Aug. 21, 2012; revision accepted Dec. 27, 2012.

For correspondence or reprints contact: Lynne L Johnson, PH 10 Center Rm. 203, 630 West 168th St., New York, NY 10032.

E-mail: lj2129@columbia.edu

Published online Apr. 26, 2013.

COPYRIGHT @ 2013 by the Society of Nuclear Medicine and Molecular Imaging, Inc.
}

with caspase staining ( $r=0.931, P=0.002)$. Conclusion: Transforming growth factor $\beta 1$-conditioned human MSCladen patches reduce myocyte apoptosis in the setting of acute infarction, and this effect can be detected by in vivo imaging with 99mTc-HYNIC annexin V.

Key Words: apoptosis; myocardial infarction; stem cells; radionuclides; engineered cardiac tissue

J Nucl Med 2013; 54:977-983

DOI: 10.2967/jnumed.112.112979

$\mathbf{T}$ ransient and low rates of cell engraftment represent a major limitation of cell therapy for the ischemic heart. The mixed results seen in the clinical setting may be a result of poor cell retention and suboptimal cell preparations (1-4). Combining cell preconditioning with factors such as transforming growth factor (TGF- $\beta 1$ ) and improving cell retention via patch delivery have been proposed as approaches for enhancing the efficacy of cell therapy $(5,6)$. In vitro models of cardiac tissue can be used as test beds for therapeutic interventions and have been developed to evaluate the comparative potential for different methods for engineering of the cells and patches to facilitate long-term survival $(7,8)$. Imaging technology allows longitudinal in vivo interrogation of paracrine effects of therapeutic cells.

Imaging modalities used in cardiovascular cell therapy trials have largely relied on measuring regional or global left ventricular (LV) function. Although in some cases these tests have demonstrated improvements in global or regional function, there is a need for more sensitive longitudinal assays that will provide insight into mechanisms that may be driving the observed improvements in cardiac function. In the acute phase of post-myocardial infarction, a host of inflammatory responses are both detrimental, by increasing cell death via apoptosis, and beneficial, by stimulating angiogenesis (9-11). The functional benefits of cell therapy with mesenchymal stem cells (MSCs) or progenitor cells have been largely attributed to the action of paracrine factors that are proangiogenic and antiapoptotic (12-17). 
Cell membrane alterations occur in cells undergoing apoptosis to allow phagocytic engulfment. Phosphatidylserine, which is normally restricted to the inner layer of the phospholipid bilayer cell membrane, is flipped to the outer bilayer. This alteration in cell membrane has been used to target apoptosis for radionuclide imaging. The naturally occurring protein annexin $\mathrm{V}$ avidly binds phosphatidylserine and can be labeled with a radiotracer for in vitro and in vivo imaging of apoptosis (18). This imaging approach has been used in experimental animal models and in clinical applications (19-21).

In this study, we hypothesized that in vivo apoptosis imaging could be used to evaluate the cardioprotective effects of a cell-delivery platform in the acute post-myocardial infarction heart. To this end, we monitored the effects of our therapeutic approach in live animals using apoptosis imaging and serial ${ }^{99 \mathrm{~m} T c-a n n e x i n ~ V ~ h y b r i d ~ S P E C T / C T ~ s c a n s . ~}$

\section{MATERIALS AND METHODS}

\section{Cardiac Constructs}

Neonatal rat ventricular myocytes (NRVMs) were suspended in fibrin hydrogel $(2 \% \mathrm{w} / \mathrm{v}$ fibrinogen, thrombin $[125 \mathrm{KU} / \mathrm{mL}])$, seeded onto Ultrafoam (Davol Inc.) collagen scaffolds $\left(16.6 \times 10^{6}\right.$ cells $/ \mathrm{cm}^{3}$ ), and cultured for $4 \mathrm{~d}$ in medium (Dulbecco modified Eagle medium, $10 \%$ fetal bovine serum, and $1 \%$ penicillin/streptomycin).

\section{In Vitro Cardiac Repair Experiments}

We established 2 groups to simulate the engraftment of a fibrin patch over cardiac constructs: fibrin alone and fibrin with encapsulated TGF- $\beta 1$-conditioned MSC (cells seeded at $1.0 \times 10^{6} / 80 \mu \mathrm{L}$ ). Fibrin patches were seeded over the beating NRVM constructs, allowed to polymerize, and placed in culture for $1 \mathrm{~d}$. At day 5 , constructs were placed under hypoxic conditions $\left(5 \% \mathrm{O}_{2}, 5 \% \mathrm{CO}_{2}\right.$, and $90 \% \mathrm{~N}_{2}$ ) and cultured for $1 \mathrm{wk}$, and samples were taken at the following 3 time points: immediately before placement in hypoxia, after $24 \mathrm{~h}$, and at $1 \mathrm{wk}$ of hypoxic culture. At the end of the experiment, each construct was paraffin-embedded, sectioned ( $5 \mu \mathrm{m}$ thick), and stained for caspase- 3 and hematoxylin and eosin (H\&E). The number of caspase-positive cells for fibrin control constructs $(n=7)$ and conditioned MSC constructs $(n=7)$ were compared.

\section{Fibrin Patch for In Vivo Studies}

TGF- $\beta 1$-conditioned MSC patches were prepared by modifying a previously reported cell-delivery approach (5). Specifically, passage-5 MSCs were conditioned with TGF- $\beta 1(0.1 \mathrm{ng} / \mathrm{mL})$ (R\&D Systems) for $4 \mathrm{~d}$, encapsulated in $2 \%$ fibrin hydrogel, loaded onto a highly porous poly(glycerol sebacate) scaffold known as Biorubber (kindly provided by Dr. Yadong Wang) to enable suturing, and cultured in TGF- $\beta 1$-supplemented medium for an additional $3 \mathrm{~d}$ before implantation (Fig. 1). Acellular control patches were constructed following the same protocol, except that the cells were not added to the fibrin hydrogel and the TGF- $\beta 1$ was not supplemented to culture medium.

\section{Animal Groups}

The animal protocol was approved by the Institutional Animal Care and Use Committee of Columbia University. Twenty-five nude male rats (T cell-deficient NIH; Charles River Laboratories) underwent left anterior descending coronary artery (LAD) occlusion. Two rats died early during surgery, and the remaining 23 (successful infarctions) were divided into 3 groups to receive the following: TGF- $\beta 1$-conditioned human MSC-laden patches (CP), infarct alone without patch (no patch [NP]), and patch alone (patch only [PO]). Twenty-four hours after myocardial infarction, all of these rats were injected with ${ }^{99 \mathrm{~m}} \mathrm{Tc}$-hydrazinonicotinamide ( ${ }^{99 \mathrm{~m}} \mathrm{Tc}-\mathrm{HYNIC}$ ) annexin $\mathrm{V}$ and ${ }^{201} \mathrm{Tl}$ and underwent dual-isotope SPECT/CT imaging. Six rats (2 per group) were sacrificed for histology and counting. The remaining 17 rats were injected and imaged on day 7, after which 1 rat from each group was sacrificed for histology. One rat had a small infarction due to distal LAD ligations and was eliminated, leaving 16 rats for data analysis with imaging on day 7 (CP, 6; NP, 5; and PO, 5) and 13 that survived to day 21 .
FIGURE 1. Tissue-engineering in vitro model system. (A) Fibrin-encapsulated TGF- $\beta 1-$ conditioned human MSCs are seeded over in vitro cardiac constructs. (B) Acellular fibrin gel is used as control. Tissue sections show immunofluorescent staining for troponin (green), caspase-3 (red), and nuclei 4',6-diamidino-2-phenylindole (blue for nuclei), after 1 and $7 \mathrm{~d}$ of in vitro culture under hypoxic conditions ( $5 \%$ oxygen). Arrow points to engrafted TGF- $\beta 1-$ conditioned MSCs. There is less caspase-3 staining (therefore less apoptosis) in cardiac constructs treated with TGF- $\beta 1-$ conditioned human MSC fibrin than in control group at both time points. (C) Caspase-3-positive cells $(5-\mu \mathrm{m}$ section) in in vitro constructs treated with acellular patches (bar on left) or TGF- $\beta 1$-conditioned MSC patches (bar on right). (D) H\&E-stained section of MSCs in $\mathrm{CP}$ delivery patch for in vivo experiments. DAPI $=$ 4',6-diamidino-2-phenylindole; $\mathrm{Tnl}=$ troponin.

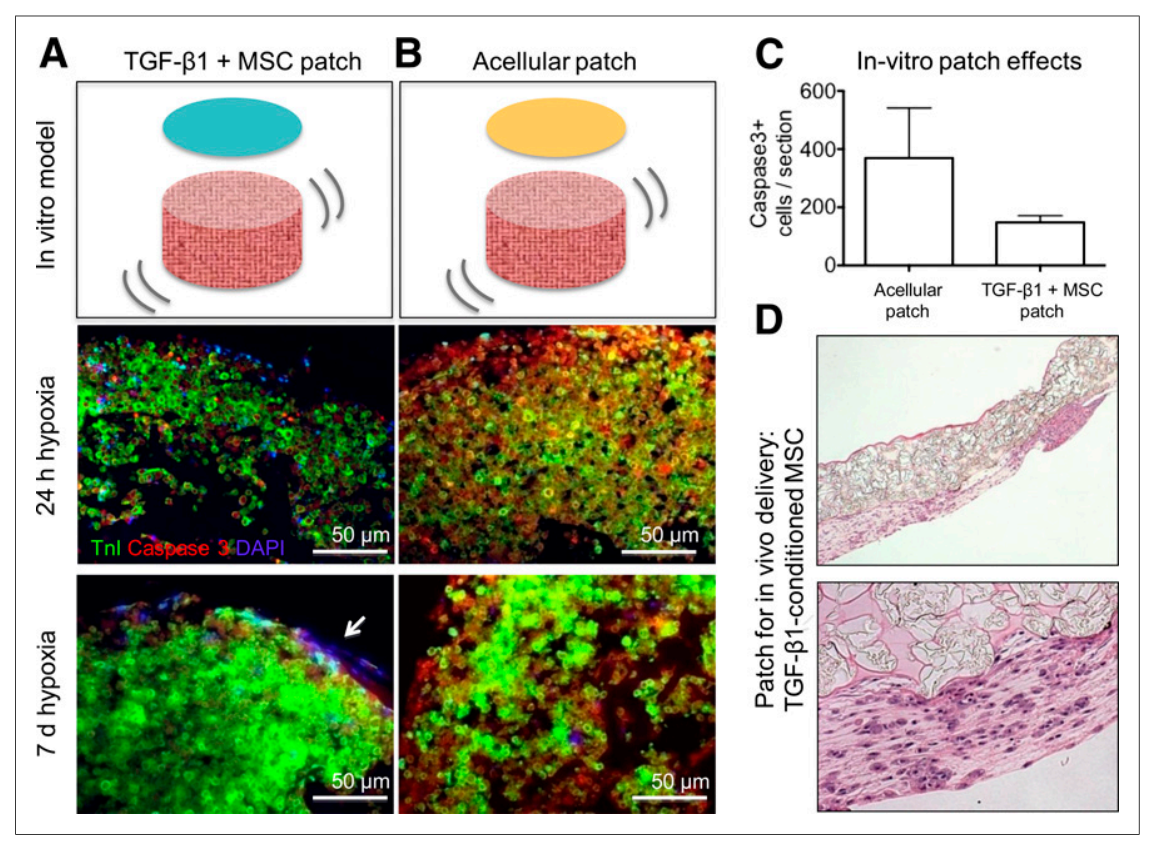




\section{Surgical Procedures}

The LAD was ligated by tying a suture around the vessel just proximal to the major branch. The tissue-engineered patch (CP or PO) was then placed over the ischemic zone and secured to the myocardium and the chest closed. A 20-gauge angiocatheter positioned before chest closure was used to evacuate intrathoracic air and fluid before cessation of mechanical ventilation and the removal of the endotracheal tube at the first sign of spontaneous respiration. Animals were evaluated at days 1, 7, and 21 .

\section{Tracer Preparation, Injection, and Imaging}

HYNIC-derivatized annexin V was provided by the National Cancer Institute repository and was labeled with ${ }^{99 \mathrm{~m}} \mathrm{Tc}$. The mean specific activity was $1.7 \pm 0.38 \mathrm{MBq} / \mu \mathrm{g}(46.3 \pm 10.3 \mu \mathrm{Ci} / \mu \mathrm{g})$, and the mean radiochemical purity of $95 \% \pm 2.6 \%$ was measured by instant thin-layer chromatography.

At days 1 and 7, rats were anesthetized and injected with $54.02 \pm$ $4.07 \mathrm{MBq}(1.46 \pm 0.11 \mathrm{mCi})$ of ${ }^{99 \mathrm{~m}} \mathrm{Tc}$-annexin $\mathrm{V}$ via a femoral venous catheter. Three hours later, each animal was injected with $11.1 \pm 0.37 \mathrm{MBq}(0.30 \pm 0.01 \mathrm{mCi})$ of ${ }^{201} \mathrm{Tl}$ and immediately underwent dual-isotope hybrid SPECT/CT imaging (Bioscan) using the rat apertures with $2.5-\mathrm{mm}$ pinholes and a resolution of 1.98 $\mathrm{mm}$ with the following imaging parameters: $30^{\circ}$ step in $360^{\circ}$ rotation using 24 projections, $60 \mathrm{~s}$ per projection, and a $256 \times$ 256 frame size with $1.0-\mathrm{mm}$ pixels. The projection data were reconstructed iteratively with an ordered-subset expectation maximization algorithm. The CT and SPECT data were fused and reconstructed using InVivoScope software (Bioscan).

At the end of scanning, the animals were either awakened and returned to animal housing or euthanized with an overdose of pentobarbital. The hearts were rapidly excised, flushed with chilled saline, and weighed. The atria were removed and ventricles sectioned transversely and counted (Wallac Wizard 1470; PerkinElmer). Tissue slices were fixed in embedding medium. The percentage of injected dose per gram $(\% \mathrm{ID} / \mathrm{g})$ of tissue was calculated from the well counter data. The radiotracer activity in the samples was corrected for background, decay time, and tissue weight.

\section{Histology}

Myocardial tissue was formalin-fixed, paraffin-embedded, and sectioned ( $5 \mu \mathrm{m}$ thick). Hearts excised at 1 and $7 \mathrm{~d}$ after myocardial infarction were stained for active caspase- 3 and $H \& E$, and hearts excised at $21 \mathrm{~d}$ were stained with $\mathrm{H} \& \mathrm{E}$ and Masson trichrome. For cellular characterization, adjacent sections were deparaffinized in xylene and treated with $0.3 \%$ hydrogen peroxide for $20 \mathrm{~min}$ to inactivate endogenous peroxidase. Tissue sections were then incubated in protein-free block (Dako) for $10 \mathrm{~min}$ to inhibit the nonspecific binding of primary antibody. Apoptotic cells were identified using activated caspase- 3 antibody (1:250; Promega). Detection was performed using horseradish peroxidase-conjugated goat antirabbit IgG (Sigma), followed by diaminobenzidine (DAB substrate kit for peroxidase; Vector Laboratories) and counterstaining with Gill hematoxylin solution. Apoptosis (caspase-positive) and fibrosis (blue staining on Masson trichrome) were quantified on all myocardial sections from each heart using Image-Pro Plus software (Media Cybernetics Inc.) and expressed as percentage of the LV area per section and averaged for the sections stained per animal. Dual immunofluorescent staining was performed with $\alpha$-active caspase- 3 (Texas red) and for cardiomyocyte sarcomeric actinin (fluorescein isothiocyanate). Areas of yellow in the merged images represented colocalization.

\section{Scan Analysis}

The volumes of the perfused LV myocardium and defect were quantified as millimeter cubed from the area of thallium uptake on the reconstructed SPECT slices using InVivoScope software and converted to mass (milligrams) assuming the specific density of the heart of $1 \mathrm{~g} / \mathrm{cm}^{3}$. Using comparable thresholds for all studies, we drew regions around thallium uptake and around the defect on sequential 5-voxel-thick coronal slices from the septum to the lateral wall, and defect areas were estimated from borders of thallium uptake (Fig. 2). The uptake of ${ }^{99 \mathrm{~m}} \mathrm{Tc}$-annexin $\mathrm{V}$ was quantified in microcuries from transverse slices using a calibration factor in the software program and divided by the decay-corrected injected dose (ID) and expressed as percentage ID (\%ID). The same scan was

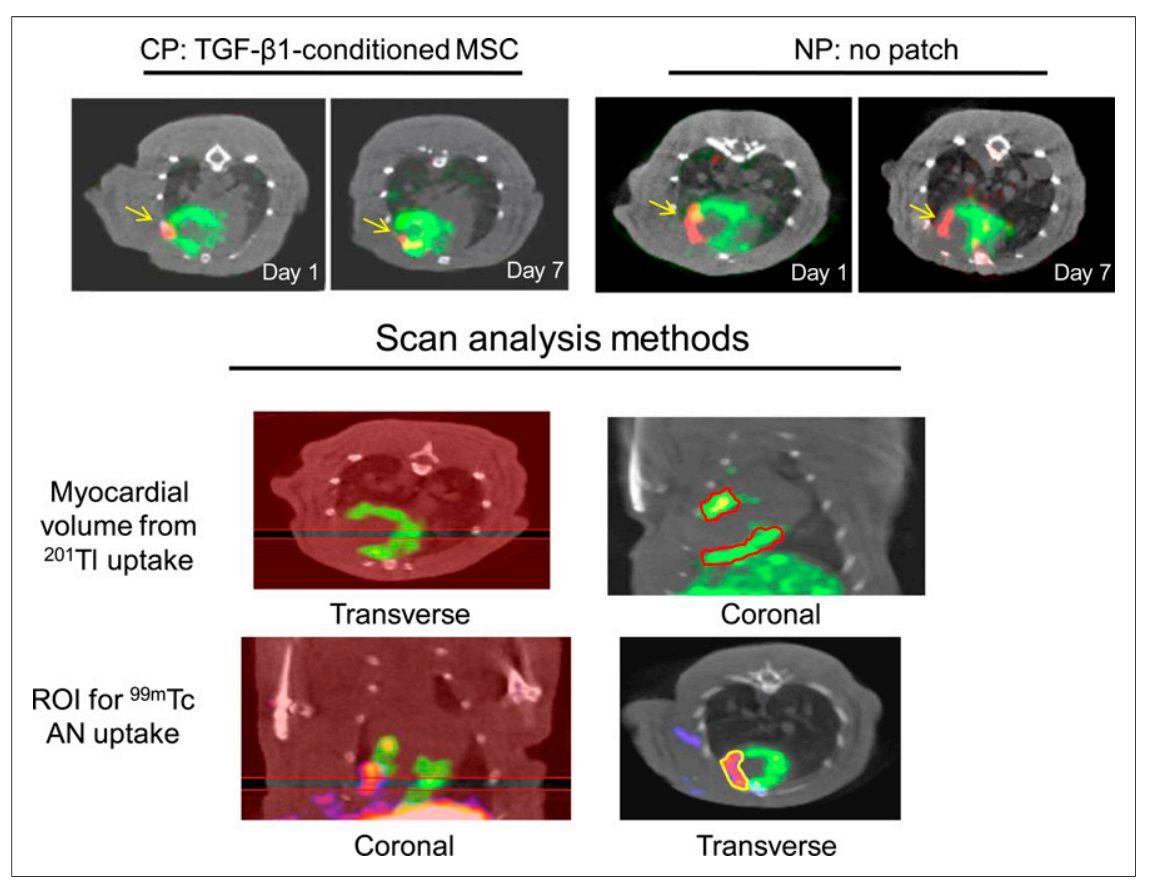

FIGURE 2. Noninvasive in vivo imaging. (Top panel) Transverse image from dual-isotope SPECT/CT scans from representative experiments from $\mathrm{CP}$ and NP groups that

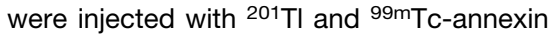
$V$ and imaged at days 1 and 7 after LAD ligation. ${ }^{201} \mathrm{TI}$ uptake in perfused myocardium is shown in green and focal uptake of $99 \mathrm{~m} T \mathrm{c}$-annexin $\mathrm{V}$ in red, with arrows pointing to this uptake. CP-treated rats showed greater decrease in $99 \mathrm{~m} T \mathrm{~T}$-annexin $\mathrm{V}$ in infarcted area between days 1 and 7 than did NP-treated rat. (Lower panel) Methods for quantification of scan data using InVivoScope software. Regions are drawn around ${ }^{201} \mathrm{Tl}$ uptake on sequential 5 -slice coronal sections from septum to lateral wall and myocardial volume measured using volume calibration. Similarly, regions are drawn around $99 \mathrm{mTc}$ uptake on sequential 4-slice sections and tracer uptake measured as millicuries, which is divided by decay-corrected ID for $\%$ ID. AN = annexin. 
analyzed twice on separate days by the same observer to determine the intraobserver reproducibility of the volume measurements and to determine the interobserver reproducibility.

\section{Statistical Analysis}

Continuous variables were expressed as mean \pm SD. Normality was assessed using the Sharpiro-Wilk W test. The equality of variances was assessed using the Levene test. Comparisons between groups were made using a paired 2-tail Student $t$ test or the MannWhitney $U$ test, as appropriate, with a $P$ value less than 0.05 denoting significance. Comparison among multiple groups was performed using ANOVA. Correlation was assessed using the Pearson product-moment correlation coefficient. Statistical analyses were performed using STATA (version 10.1; StataCorp, College Station). Inter- and intraobserver variability was assessed using the Bland-Altman method for measuring agreement, and results were expressed as bias \pm 1.96 SDs.

\section{RESULTS}

\section{In Vitro Model System}

Staining with activated caspase-3 antibody in the NRVM constructs after exposure to hypoxia showed lower activity in the TGF- $\beta 1$-conditioned MSC group. At $7 \mathrm{~d}$, the average number of caspase-positive cells per 5- $\mu \mathrm{m}$ section of NRVM scaffolds was $369 \pm 172$ for the control (fibrin patch-treated) construct and $148 \pm 23$ for the treated construct (TGF$\beta 1$-conditioned MSCs in fibrin) $(P=0.01)$ (Figs. 1A-1C).

\section{Infarct Experiments}

Sixteen rats survived surgery, were injected with radiotracer, and were imaged on days 1 and 7 and found to have an infarct size greater than $10 \%$. Nine were sacrificed at the end of either day 1 or day 7 for ex vivo counting and immunohistology. Thirteen rats survived to day 21 for histology.

\section{Reproducibility for Volume Measurements}

The intraobserver and interobserver variability for scan determinations of volumes was excellent. The limits of agreement for intraobserver variability were -10.6 to +10.2 , with a mean difference of -0.20 (confidence interval, -3.92 to 3.52). The limits of agreement for interobserver variability were -11.35 to +11.35 , with mean difference of 0.000 (confidence interval, -4.06 to 4.06 ).
TABLE 1

Defect as Percentage Myocardium

\begin{tabular}{lcc}
\hline Group & Day 1 & Day 7 \\
\hline CP & $23 \pm 6$ & $14 \pm 3$ \\
Mean \pm SD & $15-32$ & $9-17$ \\
Range & $18 \pm 4$ & $19 \pm 4$ \\
NP & $12-23$ & $15-24$ \\
Mean \pm SD & $22 \pm 5$ & $22 \pm 2$ \\
Range & $17-27$ & $20-24$ \\
PO & & \\
Mean \pm SD & 0.14 & 0.05 \\
Range & 0.80 & 0.002 \\
\hline NP vs. CP & 0.20 & 0.30 \\
\hline PO vs. CP & & \\
NP vs. PO &
\end{tabular}

\section{Volume and Defect Size}

The LV volume (mass in milligrams) did not change significantly between days 1 and 7 for any of the 3 groups: day 1, $210 \pm 40$, and day 7, $192 \pm 44$, for the CP group; day $1,201 \pm 19$, and day $2,195 \pm 23$, for the NP group; and day1, $218 \pm 38$, and day 7, $219 \pm 31$, for the PO group. There were no significant differences between mean values for defect as percentage myocardium at day 1 for NP versus $\mathrm{CP}, \mathrm{PO}$ versus $\mathrm{CP}$, or NP versus $\mathrm{PO}$, whereas for day 7 the defect size was lower in the $\mathrm{CP}$ group than in the NP $(P=$ $0.05)$ and $\mathrm{PO}$ groups $(P=0.002)$ (Tables 1 and 2$)$. The change in defect between days 1 and 7 as percentage myocardium was $-8.83 \pm 4.40$ for the $\mathrm{CP},+1.00 \pm 2.24$ for $\mathrm{NP}$, and $-0.5 \pm 4.20$ for PO groups (Fig. 3A). The difference between NP and CP was significant at a $P$ value of 0.001 and between $\mathrm{PO}$ and $\mathrm{CP}$ at 0.02 , and there was no significant difference between NP and PO groups $(P=0.51)$ (Fig. 3A).

\section{Annexin Data}

Focal uptake of ${ }^{99 \mathrm{~m}} \mathrm{Tc}$-annexin $\mathrm{V}$ was seen in the region of the ${ }^{201} \mathrm{Tl}$ defect on day 1 in all experiments (Fig. 2A). There were no significant differences between mean values for ${ }^{99 \mathrm{~m}} \mathrm{Tc}-$ annexin $\mathrm{V}$ uptake (\%ID) between NP versus CP, $\mathrm{PO}$ versus $\mathrm{CP}$, or $\mathrm{NP}$ versus $\mathrm{PO}$ for day 1 . At day 7 , focal uptake of ${ }^{99 \mathrm{~m}} \mathrm{Tc}$-annexin $\mathrm{V}$ was seen in the region of the
FIGURE 3. Imaging data. (A) Shown is reduction in ischemic defect (IS) as percentage left ventricle from day 1 to day 7 in 3 groups. Defect was reduced in area in CP group, whereas there were small changes in the 2 control groups. (B) Reduction in ${ }^{99 m} \mathrm{Tc}-$ annexinV uptake from day 1 to day 7 in 3 groups. Reduction in tracer uptake in defect region is greater in $\mathrm{CP}$ than in NP or $\mathrm{PO}$ groups, and there is no change between NP and PO groups.

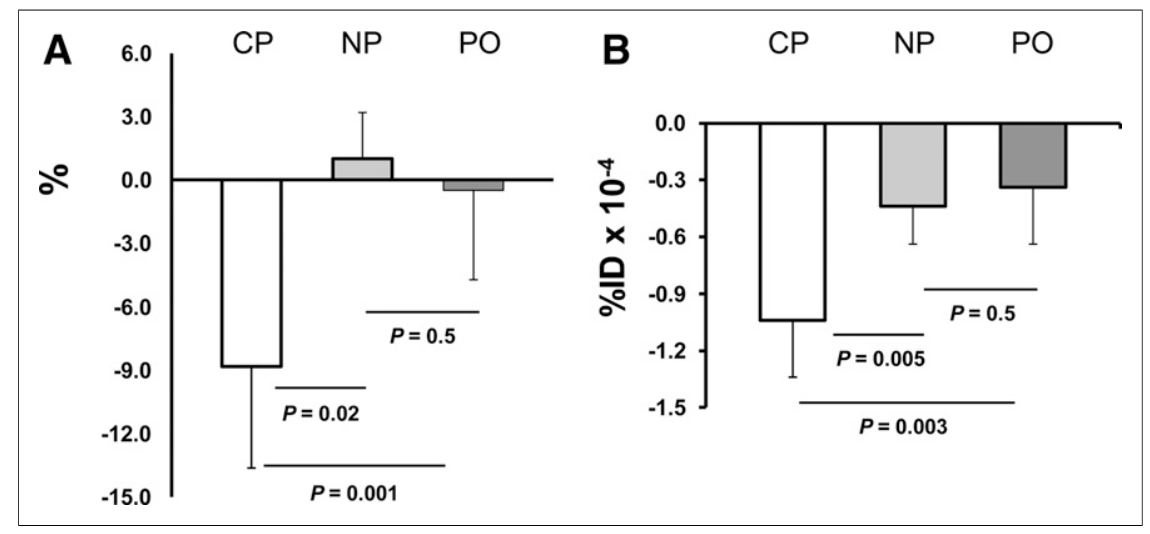


${ }^{201} \mathrm{Tl}$ defect in all but 1 experiment. Probe uptake was lower in CP than in NP groups $(P=0.04)$ and borderline lower in the PO than in $\mathrm{CP}$ groups $(P=0.08)$ (Table 2). Change in ${ }^{99 \mathrm{~m}} \mathrm{Tc}$-annexin $\mathrm{V}$ uptake (\%ID) between days 1 and 7 were $-1.04 \pm 0.28$ in the CP group, $-0.44 \pm 0.17$ in the NP group, and $-0.34 \pm 0.27$ in the $\mathrm{PO}$ groups $(P=0.003, \mathrm{NP}$ vs. $\mathrm{CP} ; P=0.005$, $\mathrm{PO}$ vs. $\mathrm{CP}$; and $P=0.5, \mathrm{NP}$ vs. $\mathrm{CP}$ ) (Fig. 3B). The uptake of ${ }^{99 \mathrm{~m}} \mathrm{Tc}$-annexin $\mathrm{V}$ measured from the scans correlated with measured values of $\% \mathrm{ID} / \mathrm{g}$ in the hearts harvested at days 1 and $7(r=0.883, P=0.03)$ (Fig. 4).

\section{Histologic Staining}

Myocardial sections from the risk region from representative animals sacrificed at days 1 and 7 stained for caspase-3 showed greater staining at day 1 than at day 7. There were more cells staining positive at day 7 in the control experiments than in the CP experiments. Dual fluorescence staining showed localization of caspase-3 to myocytes (Fig. 5).

\section{Correlations Between Imaging and Histology}

For the hearts removed at days 1 and 7, there was a significant correlation between ${ }^{99 \mathrm{~m}} \mathrm{Tc}$-annexin $\mathrm{V}$ uptake on the scans as percentage LV cross-sectional volume and caspase staining as percentage $\mathrm{LV}$ area $(r=0.92, P=$ 0.003 ) (Fig. 5). The final size of scarring at day 21 was compared with the defect size at day 1 to account for differences in initial size. The change in defect size as percentage myocardium from day 1 to day 21 was $29 \%$ in the CP group and $11 \%$ in the control groups $(P=0.02)$ (Table 1$)$. Histologic sections from hearts harvested at day 21 from experiments with patch and without patch are shown in Figure 6.

\section{DISCUSSION}

We investigated the hypothesis that conditioned human MSCs embedded in an engineered patch would protect the myocardium by reducing apoptosis of the underlying cardiac myocytes and thereby reduce infarct size. We first

TABLE 2

99mTc-HYNIC Annexin V Uptake as \%ID $\left(\times 10^{-4}\right)$

\begin{tabular}{ccc}
\hline Group & Day 1 & Day 7 \\
\hline CP & $1.21 \pm 0.40$ & $0.18 \pm 0.13$ \\
Mean \pm SD & $0.66-1.80$ & $0.00-0.40$ \\
Range & & \\
NP & $0.84 \pm 0.21$ & $0.37 \pm 0.12$ \\
Mean \pm SD & $0.60-1.10$ & $0.15-0.46$ \\
Range & $0.80 \pm 0.46$ & $0.46 \pm 0.31$ \\
PO & $0.42-1.40$ & $20-24$ \\
Mean \pm SD & & \\
Range & 0.10 & 0.04 \\
$P \quad$ NP vs. CP & 0.17 & 0.08 \\
PO vs. CP & 0.90 & 0.60 \\
NP vs. PO & & \\
\hline
\end{tabular}

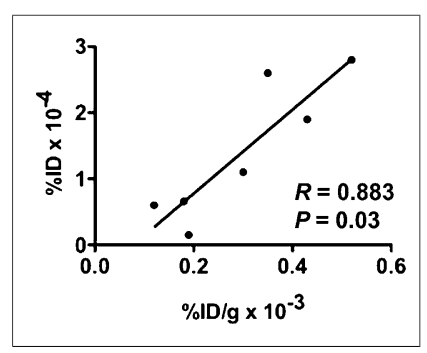

FIGURE 4. Correlation between values for ${ }^{99 m}$ Tc-annexin $\mathrm{V}$ uptake as $\% \mathrm{ID} \times 10^{-4}$ vs. ex vivo $\% \mathrm{ID} / \mathrm{g} \times 10^{-3}$.

conducted in vitro studies (using engineered cardiac constructs in the setting of hypoxia), and then in vivo studies using a nude rat model of myocardial infarction, in which an engineered composite polymer patch containing TGF$\beta 1$-conditioned human MSCs was applied over the ischemic cardiac tissue. The beating constructs treated with TGF- $\beta 1-$ conditioned MSC patches and exposed to hypoxia were more resistant to apoptosis than the controls. In the in vivo rat infarct model, hearts grafted with patches embedded with TGF- $\beta 1-$ conditioned human MSCs showed a greater reduction in defect sizes between days 1 and 7 after LAD ligation in the $\mathrm{CP}$ group than in controls. There was a greater reduction in uptake of ${ }^{99 \mathrm{~m}} \mathrm{Tc}-\mathrm{HYNIC}$ annexin $\mathrm{V}$ between days 1 and 7 in CP than in the PO and NP groups. There were no statistically significant differences in ${ }^{99 \mathrm{~m} T c-H Y N I C}$ annexin $\mathrm{V}$ uptake on day 1 among the 3 groups, whereas there was significant or borderline significantly lower uptake on day 7 in the CP, as compared with the PO and NP groups. These differences suggest that the beneficial effect of TGF$\beta 1$-conditioned human MSCs to reduce defect size was achieved through the reduction of apoptosis.

We have previously shown that the release of cardioprotective factors can be augmented by the synergistic effects of TGF- $\beta 1$ conditioning and hypoxia (5). Biochemical signaling from TGF- $\beta 1$ combined with a fibrin matrix promotes a profile favorable for mesenchymal progenitor cells to respond to endogenous gradients and migrate into the infarct bed (5). At the injury site, these cells contribute to cytokine secretion, attract endogenous repair cells, promote angio- and arteriogenesis, and ultimately preserve myocardial function. We hypothesized that these same factors may play an additional role in protecting the host myocardium in the postinfarcted heart and sought to measure any beneficial effects by quantitative apoptosis imaging.

Radiolabeled annexin $\mathrm{V}$ has been used to identify and quantify cell death in myocardial infarction in experimental studies and in patients (19-22). Both apoptosis and necrosis of cardiomyocytes occur in the ischemic zone after coronary artery occlusion, with apoptosis being the predominant process and apoptotic-to-necrotic cell ratios in the order of 40 to $1(11,23)$. Necrosis is characterized by the loss of homeostasis, cell swelling, and early cell membrane rupture, and apoptosis is a regulated process requiring energy and characterized by cell shrinkage, condensation of chromatin, and budding of the cell membrane into vesicles containing cellular organelles called apoptotic bodies (10). 
FIGURE 5. (A) Graph shows correlation between ${ }^{99 \mathrm{~m} T c-a n n e x i n ~} \mathrm{~V}$ uptake as percentage area of left ventricle (from regionof-interest analysis) and percentage area of caspase staining from quantitative immunostaining of tissue from animals that were sacrificed after scans. (B) Histologic sections are shown for apoptosis staining light microscopy sections of myocardium in risk region from control rat at day 1 and day 7 and from CP rat at day 7 (left) and immunofluorescence-stained sections from a day 1 $\mathrm{CP}$ experiment with staining for sarcomeric $\alpha$-actinin in red, caspase- 3 in green, and two merged in yellow (right). At day 1 , there are more positive-stained cells than at day 7 , and at day 7 there are fewer positive-stained cells for CP than for NP experiments. Caspase- 3 colocalizes with myocytes. $\mathrm{AN}=$ annexin.

Apoptotic cells are seen predominantly at the borders of infarct border zones, and therefore the extent of apoptosis determines infarct size $(24,25)$.

Annexin $\mathrm{V}$ is a naturally occurring peptide found in myocardial cells and may have a role in interstitial fibrosis and myocardial remodeling (26). Transient sarcolemmal phosphatidylserine expression has been observed during periods of brief ischemia, with the capacity of the cells to return to normal functioning with the restoration of oxygen suggesting that uptake of ${ }^{99 \mathrm{~m}} \mathrm{Tc}$-annexinV is not specific for irreversible programmed cell death and that some myocytes binding this tracer may survive (27). In the model of complete coronary artery ligation used in this study, ischemia was not transient, and therefore it is likely that cells binding ${ }^{99 \mathrm{~m}} \mathrm{Tc}$-annexin $\mathrm{V}$ are undergoing irreversible cell death. Although it has been postulated that ${ }^{99 \mathrm{~m}} \mathrm{Tc}$-annexin $\mathrm{V}$ can bind necrotic and apoptotic cells, the experimental evidence supports the premise that uptake localizes to areas of apoptotic cells exclusively up to $4.5 \mathrm{~h}$ after occlusion (25). The histologic staining we performed suggests that the primary cell types undergoing apoptosis at both days 1 and 7 are myocytes. However, during the early infarct healing period there is an ongoing dynamic interplay of many processes, including inflammation and angiogenesis, that involves several cell types, and the uptake of ${ }^{99 \mathrm{~m}} \mathrm{Tc}$-annexinV probe on scans represents the sum total of programmed cell death.

On the basis of these findings from in vitro cardiacengineered scaffolds and previously published work (5), we constructed a stem cell-delivery platform to be used in an acute infarct model and hypothesized that the cardioprotective effect by reducing apoptosis can be quantified on in vivo SPECT imaging. Although the apoptotic activity seen $24 \mathrm{~h}$ after infarction was greater at day 1 than at day 7 in all groups, only the MSC group had a significantly greater reduction in apoptotic activity over $1 \mathrm{wk}$, accompanied by reduced ischemic defect size, and reduced fibrosis at 21 d. It is postulated that these observed beneficial effects are due to the release of paracrine factors after stem cell engraftment and augmented by pharmacologic and physiologic conditioning $(28,29)$ or through transgenic approaches $(30-32)$.

\section{CONCLUSION}

TGF- $\beta 1$-conditioned human MSC patches reduce myocyte apoptosis in both an in vitro model of cardiac repair and in the in vivo setting of acute myocardial infarction. This antiapoptotic effect can be detected by in vivo imaging with ${ }^{99 \mathrm{~m}} \mathrm{Tc}-\mathrm{HYNIC}$ annexin $\mathrm{V}$ and presents a method for noninvasive and longitudinal monitoring of cell-based interventions in the heart. This noninvasive imaging approach has potential as a tool to document beneficial effects of stem cell therapy that are upstream of changes in regional or global cardiac function. Although this approach to delivering stem cells is not practical in an acute myocardial infarction setting in patients. It may be feasible to implant these patches in patients with chronic ischemic heart disease at the time of surgery to prevent ongoing myocyte death in areas of the myocardium that cannot be revascularized. Dual-isotope SPECT/CT imaging, a standard clinical tool, may thus serve as a reliable measure for the progression of patients undergoing cell therapy, as a metric for therapeutic effect.

\section{DISCLOSURE}

The costs of publication of this article were defrayed in part by the payment of page charges. Therefore, and solely

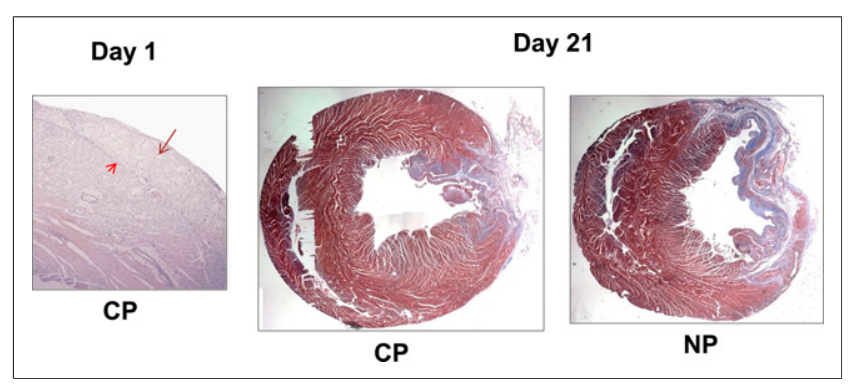

FIGURE 6. Effects of patch implantation on fibrosis in infarct bed. (Left) H\&E-stained section from day 1 CP experiment showing position of patch over infarct zone. Longer arrow points to patch and shorter arrow to interface between patch and myocardium. (Middle and right) Trichrome myocardial sections showing normal myocardium in red-purple and fibrosis in blue. In these 2 representative rats, histology shows a small area of scarring in CP group and larger area of scarring in NP group. 
to indicate this fact, this article is hereby marked "advertisement" in accordance with 18 USC section 1734. This study was supported in part by R01 HL076485, P41 EB002520, C026449, and R21HL 108668. No other potential conflict of interest relevant to this article was reported.

\section{ACKNOWLEDGMENT}

The samples of Biorubber polymer sponge were kindly provided by Dr. Yadong Wang.

\section{REFERENCES}

1. Assmus B, Honold J, Schachinger V, et al. Transcoronary transplantation of progenitor cells after myocardial infarction. $N$ Engl J Med. 2006;355:1222-1232.

2. Assmus B, Schachinger V, Teupe C, et al. Transplantation of progenitor cells and regeneration enhancement in acute myocardial infarction (TOPCARE-AMI). Circulation. 2002;106:3009-3017.

3. Bartunek J, Vanderheyden M, Vandekerckhove B, et al. Intracoronary injection of CD133-positive enriched bone marrow progenitor cells promotes cardiac recovery after recent myocardial infarction: feasibility and safety. Circulation. 2005;112(9 suppl):I178-I183.

4. Engelmann MG, Theiss HD, Hennig-Theiss C, et al. Autologous bone marrow stem cell mobilization induced by granulocyte colony-stimulating factor after subacute ST-segment elevation myocardial infarction undergoing late revascularization: final results from the G-CSF-STEMI (Granulocyte Colony-Stimulating Factor ST-Segment Elevation Myocardial Infarction) trial. J Am Coll Cardiol. 2006;48:1712-1721.

5. Godier-Furnemont AF, Martens TP, Koeckert MS, et al. Composite scaffold provides a cell delivery platform for cardiovascular repair. Proc Natl Acad Sci U S A. 2011;108:7974-7979.

6. Zimmermann WH, Melnychenko I, Wasmeier G, et al. Engineered heart tissue grafts improve systolic and diastolic function in infarcted rat hearts. Nat Med. 2006;12:452-458.

7. Hansen A, Eder A, Bonstrup M, et al. Development of a drug screening platform based on engineered heart tissue. Circ Res. 2010;107:35-44.

8. Song H, Yoon C, Kattman SJ, et al. Interrogating functional integration between injected pluripotent stem cell-derived cells and surrogate cardiac tissue. Proc Natl Acad Sci U S A. 2010;107:3329-3334.

9. Gwechenberger M, Mendoza LH, Youker KA, et al. Cardiac myocytes produce interleukin-6 in culture and in viable border zone of reperfused infarctions. Circulation. 1999;99:546-551.

10. James TN. The variable morphological coexistence of apoptosis and necrosis in human myocardial infarction: significance for understanding its pathogenesis, clinical course, diagnosis and prognosis. Coron Artery Dis. 1998;9:291-307.

11. Kajstura J, Cheng W, Reiss K, et al. Apoptotic and necrotic myocyte cell deaths are independent contributing variables of infarct size in rats. Lab Invest. 1996; 74:86-107.

12. Burchfield JS, Dimmeler S. Role of paracrine factors in stem and progenitor cell mediated cardiac repair and tissue fibrosis. Fibrogenesis Tissue Repair. 2008; $1: 4$.

13. Gnecchi M, Zhang Z, Ni A, Dzau VJ. Paracrine mechanisms in adult stem cell signaling and therapy. Circ Res. 2008;103:1204-1219.
14. Kocher AA, Schuster MD, Szabolcs MJ, et al. Neovascularization of ischemic myocardium by human bone-marrow-derived angioblasts prevents cardiomyocyte apoptosis, reduces remodeling and improves cardiac function. Nat Med. 2001;7:430-436.

15. Mirotsou M, Jayawardena TM, Schmeckpeper J, Gnecchi M, Dzau VJ. Paracrine mechanisms of stem cell reparative and regenerative actions in the heart. $J \mathrm{Mol}$ Cell Cardiol. 2011;50:280-289.

16. Payne TR, Oshima H, Okada M, et al. A relationship between vascular endothelial growth factor, angiogenesis, and cardiac repair after muscle stem cell transplantation into ischemic hearts. J Am Coll Cardiol. 2007;50:1677-1684.

17. Vandervelde S, van Luyn MJ, Tio RA, Harmsen MC. Signaling factors in stem cell-mediated repair of infarcted myocardium. J Mol Cell Cardiol. 2005;39:363-376.

18. Blankenberg FG, Katsikis PD, Tait JF, et al. In vivo detection and imaging of phosphatidylserine expression during programmed cell death. Proc Natl Acad Sci USA. 1998;95:6349-6354.

19. Dumont EA, Hofstra L, van Heerde WL, et al. Cardiomyocyte death induced by myocardial ischemia and reperfusion: measurement with recombinant human annexin-V in a mouse model. Circulation. 2000;102:1564-1568.

20. Hofstra L, Liem IH, Dumont EA, et al. Visualisation of cell death in vivo in patients with acute myocardial infarction. Lancet. 2000;356:209-212.

21. Wolters SL, Corsten MF, Reutelingsperger CP, Narula J, Hofstra L. Cardiovascular molecular imaging of apoptosis. Eur J Nucl Med Mol Imaging. 2007;34 (suppl 1):S86-S98.

22. Nunes SS, Song H, Chiang CK, Radisic M. Stem cell-based cardiac tissue engineering. J Cardiovasc Transl Res. 2011;4:592-602.

23. Saraste A, Pulkki K, Kallajoki M, Henriksen K, Parvinen M, Voipio-Pulkki LM. Apoptosis in human acute myocardial infarction. Circulation. 1997;95:320-323.

24. Krijnen PA, Nijmeijer R, Meijer CJ, Visser CA, Hack CE, Niessen HW. Apoptosis in myocardial ischaemia and infarction. J Clin Pathol. 2002;55:801-811.

25. Taki J, Higuchi T, Kawashima A, et al. Detection of cardiomyocyte death in a rat model of ischemia and reperfusion using ${ }^{99 \mathrm{~m}} \mathrm{Tc}-l a b e l e d$ annexin V. J Nucl Med. 2004;45:1536-1541.

26. Trouvé P, Legot S, Belikova I, et al. Localization and quantitation of cardiac annexins II, V, and VI in hypertensive guinea pigs. Am J Physiol. 1999;276: H1159-H1166.

27. Strauss HW, Narula J, Blankenberg FG. Radioimaging to identify myocardial cell death and probably injury. Lancet. 2000;356:180-181.

28. Hu X, Yu SP, Fraser JL, et al. Transplantation of hypoxia-preconditioned mesenchymal stem cells improves infarcted heart function via enhanced survival of implanted cells and angiogenesis. J Thorac Cardiovasc Surg. 2008;135:799-808.

29. Niagara MI, Haider H, Jiang S, Ashraf M. Pharmacologically preconditioned skeletal myoblasts are resistant to oxidative stress and promote angiomyogenesis via release of paracrine factors in the infarcted heart. Circ Res. 2007;100: 545-555.

30. Mangi AA, Noiseux N, Kong D, et al. Mesenchymal stem cells modified with Akt prevent remodeling and restore performance of infarcted hearts. Nat Med. 2003;9:1195-1201.

31. Noiseux N, Gnecchi M, Lopez-Ilasaca M, et al. Mesenchymal stem cells overexpressing Akt dramatically repair infarcted myocardium and improve cardiac function despite infrequent cellular fusion or differentiation. Mol Ther. 2006; 14:840-850.

32. Templin C, Kotlarz D, Faulhaber J, et al. Ex vivo expanded hematopoietic progenitor cells improve cardiac function after myocardial infarction: role of betacatenin transduction and cell dose. J Mol Cell Cardiol. 2008;45:394-403.

33. Kietselaer BL, Reutelingsperger CP, Boersma HH, et al. Noninvasive detection of programmed cell loss with ${ }^{99 \mathrm{~m}} \mathrm{Tc}$-labeled annexin A5 in heart failure. $\mathrm{J} \mathrm{Nucl}$ Med. 2007;48:562-567. 